\title{
Symmetry and optical properties of wurtzite nanostructures with the $c$-axis in the layer plane ${ }^{1}$
}

\author{
(C) P. Tronc*, P. Vendéguès \\ * Laboratoire d'Optique Physique, Ecole Supérieure de Physique et Chimie Industrielles, \\ 75005 Paris, France \\ CRHEA-CNRS, \\ 06560 Valbonne, France \\ E-mail: tronc@optique.espci.fr
}

\begin{abstract}
In wurtzite-based quantum wells and superlattices with the $c$-axis parallel to the layer plane, the plane is parallel to either a summetry plane of the wurtzite lattice (type I structures, the $\langle 11 \overline{2} 0\rangle$ growth direction) or a glide plane containing the $c$-axis (type II structures, the $\langle 10 \overline{1} 0\rangle$ growth direction). In both cases, the space symmetry of the structure depends on the parity of the number of monolayers within the slab(s). The point symmetry is $C_{2 v}$ except for the type II structures with odd monolayer number(s). The latter structures have the $\sigma_{v}$ point symmetry and can present a built-in electric field. Quite different selection rules, depending on the structure symmetry, govern electron optical transitions and exciton radiative recombination.
\end{abstract}

This work has been supported by the 11156RF and 16254YE grants from the Ministère des Affaires Etrangères (France).

PACS: 02.20.-a, 61.46.-w, 78.67.-n

\section{Introduction}

The wurtzite lattice $\left(C_{6 v}^{4}\right.$ non-symmorphic space group) has a threefold rotation axis and a $6_{3}$ improper rotation (screw) axis. Both axes are parallel to the $c$-direction but do not coincide one with the other. In addition, the $6_{3}$ screw axis is also a threefold rotation axis. Whereas atoms of the lattice lie on the threefold axes, the $6_{3}$ axes do not bear atoms. The three symmetry planes are parallel to the $c$-axis and each of the three glide planes parallel to the $c$-axis is perpendicular to one of the symmetry planes (see Figure). The wurtzite lattice is a polar one. The nanostructures grown along the $c$-axis direction present a huge built-in electric field arising from the piezoelectric effect and the difference in spontaneous polarizability between the well and barrier materials. The field reduces the electron and hole eigenenergies (quantum confined Stark effect) hence the radiative recombination energies. For wide enough, the ground transition can be less in energy than the well-material band gap. In addition, the field spatially separates electrons from holes thus reducing the exciton binding energy and the oscillator strength for radiative recombination. Such effects have been evidenced experimentally and computed numerically in numerous papers (see, for examples, Ref. [1-3] for GaN-based quantum wells (QWs) and Ref. [4] for $\mathrm{ZnO}$-based ones). In order to cancel the built-in field or at least to reduce its strength, structures have been grown with the $c$-axis in the layer plane. Wurtzite heterostructures like QWs or superlattices (SLs) can be grown with the layer plane being parallel to a symmetry plane (type I heterostructure,

\footnotetext{
1 Доклад на XIII Феофиловском симпозиуме „Спектроскопия кристаллов, активированных редкоземельными ионами и ионами переходных металлов“ (Иркутск, июль 2007 г.).

Основная часть докладов опубликована в выпуске 9 журнала „Физика твердого тела“ за 2008 г.
}

the $\langle 11 \overline{2} 0\rangle$ growth direction) or to one of the three glide planes mentionned above (type II heterostructure, $\langle 10 \overline{1} 0\rangle$ growth direction). The growth of wurtzite III-nitride or $\mathrm{ZnO}$ heterostructures along the $\langle 11 \overline{2} 0\rangle$ direction has already been achieved using substrates as (1120) $6 H$-SiC [5] and 4H-SiC [6], (0112) $\mathrm{LiTaO}_{3}$ [7] or (1102) sapphire [8], (100) $\gamma-\mathrm{LiAlO}_{2}$ [9], (1010) $6 H$-SiC [10], or even $(10 \overline{1} 0)$ $\mathrm{GaN}$ [11] have been used to obtain growth along $\langle 10 \overline{1} 0\rangle$. Hereafter we consider heterostructures whose well and barrier materials are stochiometric binary compounds with common anion or cation as for example $(\mathrm{GaN})_{m} / \mathrm{AlN}$ QWs

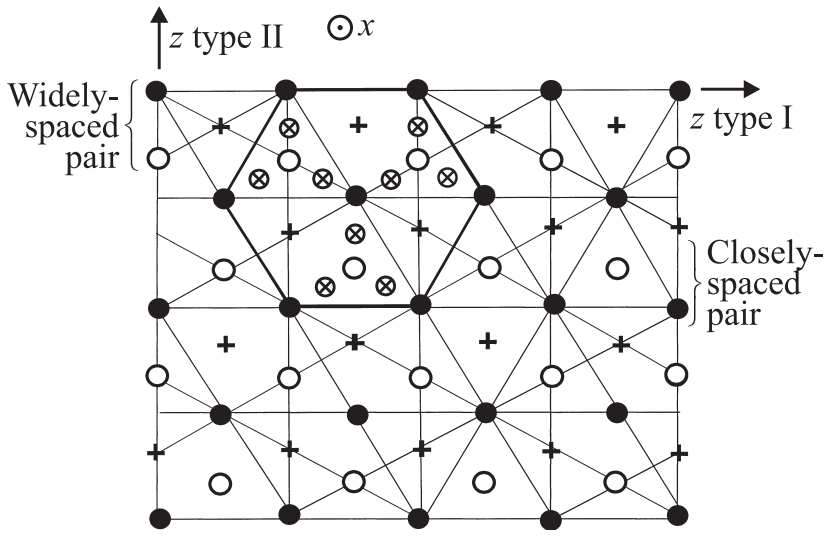

Structure of the $A B$ wurtzite lattice with the $c$-axis ( $x$-axis) perpendicular to the sheet. Blak dot: $A$ (or $B$ ) site within the $x=0$ plane. Open dot: site with an atom of the same species as within the $x=0$ plane but licated within the first adjacent layer. Atoms to the other species are not shown. Each atomic site is located on a three-fold rotation axis perpendicular to the $x=0$ plane. Cross: location of a $6_{3}$ screw axis perpendicular to the $x=0$ plane. Crossed dot: location (restricted to an hexagon) of a $21_{1}$ screw axis perpendicular to the $x=0$ plane. The $z$-axes for type I and type II structures are indicated. 
or $(\mathrm{GaN})_{m}(\mathrm{AlN})_{n}$ SLs (ZnO-based structures have also been grown). Whereas the monolayers are regularly spaced in type I heterostructures, they form closely-spaced pairs in type II heterostructures (see Figure).

\section{Symmetry analysis}

When analysing the QW and SL structures, we adopt the approximation that the atoms are on the sites of a wurtzite lattice with lattice constants being averages between the lattice parameters of well and barrier materials. Taking this approximation into account, the coordinates of all the atoms in the lattice are well defined and one can determine both the space group and the atomic arrangement over the Wyckoff positions for arbitrary numbers of monolayers $m$ and $n$. The space symmetry of QWs is described by layer groups (hereafter the labeling of layer groups follows Ref. [12]) whereas that of SLs is described by space groups. In our notations, the $x$-axis coincides with the $c$-axis of the wurtzite lattice and the $z$ direction is perpendicular to the layer plane. Each layer group has the same symmetry operations, except the translations along the $z$-axis, as one particular three-dimensional threeperiodic space group that will be labeled hereafter as the corresponding group [13]. The correspondence between layer groups and three-dimensional three-periodic space groups is provided in Ref. [14]. The two-dimensional Brillouin Zone (BZ) of the layer group coincides with the $\left(k_{x}, k_{y}\right)$ restriction of the $\mathrm{BZ}$ of the corresponding threedimensional three-periodic space group and presents the same symmetry properties as the restriction. In particular, the layer group and its corresponding group have the same point symmetry. The irreducible representations (IRs) of the little group of the $\left(k_{x}, k_{y}\right)$ wavevector can be taken directly from the tables of the IRs of the corresponding three-dimensional three-periodic space group. The optical selection rules can be established using the conventional procedure. In particular, the $\left(k_{x}, k_{y}\right)$ wavevector has to be kept in a direct transition. Within the two-dimensional $\mathrm{Bz}$, the optical selection rules are the same as for the corresponding space group in the $\left(k_{x}, k_{y}\right)$ plane.

1) Except for the type II QWs with odd values of $m$, the lattice of any QW is a primitive orthorhombic/rectangular and presents a 21 screw axis parallel to the $x$-axis (see Fugure). In addition, one has the following symmetry elements.

a) In type I QWs, a glide plane parallel to $(x, z)$ with improper translation parallel to $x$. For even values of $m$, there is also a glide plane parallel to $(x, y)$ with improper translation parallel to $y$, the layer group being $L 33\left(p b 2{ }_{1} a\right)$ and the corresponding group $C_{2 v}^{5}$ and for odd values of $m$, a symmetry plane parallel to $(x, y)$ the layer group being $L$ $29\left(p b 2_{1} m\right)$ and the corresponding group $C_{2 v}^{2}$.

b) The structure of type II QWs with even values of $m$ is made of either closely-spaced pairs of monolayers only of widely-spaced pairs of monolayers only (see Fugure). In both cases, there are a symmetry plane parallel to $(x, z)$ and a glide plane parallel to $(x, y)$. In the former case, the improper translation is diagonal for $m=2(2 M-1)$ and parallel to $x$ for $m=4 N$, where $M$ and $N$ are positive integers (for example, for $m=2$, the improper translation is diagonal). In the latter case, the direction of the improper translation for a given value of $m$ is exchanged in comparison with that occurring in the former case. The layer group and the corresponding group are $L 28\left(p m 2_{1} b\right)$ and $C_{2 v}^{2}$, respectively, when the improper translation is parallel to $x$, and $L 32\left(p m 2_{1} n\right)$ and $C_{2 v}^{7}$, respectively, when it is diagonal.

For type II QWs with odd values of $m$, the lattice is monoclinic/rectangular and the only symmetry element is a symmetry plane parallel to $(x, z)$. The layer group is $L 11$ ( $p m 11)$ and the corresponding group is $C_{s}^{1}$.

2) The symmetry of SLs includes the translational symmetry along the $z$-direction. It implies that the SL period involves an integer number of the wurtzite-lattice period in the growth direction. Therefore $m+n$ should be equal to $2 P$ and $4 Q$ for type I and type II SLs, respectively, where $P$ and $Q$ are positive integers. It follows that $m$ and $n$ should have the same parity. There is no other limitation for type I SLs. For type II SLs, the couples of $(m, n)$ values (for example $(2,4)$ or $(3,3)$ ) that are not such as $m+n$ is an integer multiple of 4 are forbidden since precluding the SL translational symmetry along the $z$ direction. The SL symmetry is described by space groups. The type II SLs with odd values of $m$ and $n$ have only a single point symmetry element, i. e., a symmetry plane parallel to $(x, z)$. Their space symmetry is described by the $C_{s}^{1}$ space group. For other SLs, there is a $2_{1}$ screw axis parallel to the $x$-axis (see Figure). In addition, one has the following symmetry elements.

a) In type I SLs, a glide plane parallel to $(x, z)$ with improper translation parallel to $x$. For even values of $m$ and $n$, there is also a glide plane parallel to $(x, y)$ with improper translation parallel to $y$, the space group being $C_{2 v}^{5}$ and for odd values of $m$ and $n$, a symmetry plane parallel to $(x, y)$ the space group being $C_{2 v}^{2}$.

b) The structure of type II SLs with even values of $m$ and $n$ is made of either closely-spaced pairs of monolayers only of widely-spaced pairs of monolayers only (see Figure). In both cases, there are a symmetry plane parallel to $(x, z)$ and a glide plane parallel to $(x, y)$. In the former case, the direction of the improper translation can be deduced from those in single QWs with the same numbers of monolayers as in the SL well and barrier slabs using relations (1)

$$
\begin{gathered}
\text { (diagonal }) \times(\text { diagonal }) \rightarrow(\text { diagonal }), \\
(\| x) \times(\| x) \rightarrow(\| x),
\end{gathered}
$$

where the direction of the SL improper translation appears in the right side of the relations. For example, for $m=n=2$, the improper translation is diagonal. Note that the well and barrier slabs cannot have different improper-translation directions. Indeed $m+n$ would be $[4(M+N)-2]$ that cannot be equal to $4 Q$. The relations (1) also hold in the latter case. The SLs with the 
Table 1. Space symmetries of the various structures

\begin{tabular}{|c|c|c|}
\hline Structure & Space symmetry & $\begin{array}{l}\text { Corresponding } \\
\text { group }\end{array}$ \\
\hline $\begin{array}{l}\text { QW } \\
\text { Type I, even } m \\
\text { odd } m \\
\text { Type II, even } m \text { (see text) } \\
\text { odd } m\end{array}$ & $\begin{array}{c}L 33\left(p b 2_{1} a\right) \\
L 29\left(p b 2{ }_{1} m\right) \\
L 28\left(p m 2_{1} b\right) \\
\text { or } L 32\left(p m 2_{1} n\right) \\
L 11(p m 11)\end{array}$ & $\begin{array}{c}C_{2 v}^{5}\left(P c a 2_{1}\right) \\
C_{2 v}^{2}\left(P m c 2_{1}\right) \\
C_{2 v}^{2}\left(P m c 2_{1}\right) \\
C_{2 v}^{7}\left(P m n 2_{1}\right) \\
C_{s}^{1}(P 1 m 1)\end{array}$ \\
\hline $\begin{array}{l}\text { SL } \\
\text { Type I }(m+n=2 P) \text {, even } m \text { and } n \\
\quad \text { odd } m \text { and } n \\
\text { Type II }(m+n=4 Q) \text {, even } m \text { and } n \text { (see text) } \\
\quad \text { odd } m \text { and } n\end{array}$ & $\begin{array}{c}C_{2 v}^{5}\left(P c a 2_{1}\right) \\
C_{2 v}^{2}\left(P m c 2_{1}\right) \\
C_{2 v}^{2}\left(P m c 2_{1}\right) \\
\text { or } C_{2 v}^{7}\left(P m n 2_{1}\right) \\
\quad C_{s}^{1}(P 1 m 1)\end{array}$ & \\
\hline
\end{tabular}

improper translation parallel to $x$ have the $C_{2 v}^{2}$ space group whereas those with the diagonal translation have the $C_{2 v}^{7}$ group. The results concerning both QWs and SLs are displayed in Table 1 . It is worth noticing that the $2_{1}$ screw axis of the structures with the $C_{2 v}$ point symmetry is in any case parallel to the $c$-axis of the wurtzite lattice.

3) The site-symmetry of an atom in the lattice is $C_{1}$ (no symmetry) except when the atom is located within a symmetry plane. In the latter case the site-symmetry includes the plane and is described by the $C_{s}$ group (the $\sigma_{v}$ or $\sigma_{h}$ group depending the symmetry plane is perpendicular or parallel to the layer plane).

\section{Dipolar optical selection rules and exciton radiative recombination}

Bulk hexagonal $\mathrm{GaN}$ and $\mathrm{ZnO}$ are direct-gap semiconductors (at the $\Gamma$ point). The symmetries of the lower conduction band and of the three upper valence bands (the latters in increasing energy ordering) in $\mathrm{GaN}$ are described by the $\Gamma_{7}, \Gamma_{9}, \Gamma_{7}$, and $\Gamma_{7}$ double-valued IRs of the $C_{6 v}^{4}$ space group, respectively. Hereafter the labeling of space group IRs follows Ref. [15]. Note that when the spin-orbit interaction (SOI) is not taken into account the symmetries of the lower conduction band and of the upper valence bands (they are only two) are described by the $\Gamma_{1}, \Gamma_{6}$, and $\Gamma_{1}$ single-valued IRs of the $C_{6 v}^{4}$ space group, respectively [16]. Taking into account the SOI, the $\Gamma_{1}$ IR transforms into $\Gamma_{7}$ whereas $\Gamma_{6}$ splits into $\Gamma_{7}+\Gamma_{9}$. In $\mathrm{ZnO}$, the symmetries of the lower conduction band and of the three upper valence bands are the same as in $\mathrm{GaN}$ but the ordering in energy of the valence bands is perhaps defferent. It seems reasonable to assume that the nanostructures considered in the present paper are, like bulk $\mathrm{GaN}$ and $\mathrm{ZnO}$, direct-gap semiconductors at the $\Gamma$ point, except for perhaps structures with very thin slabs (few monolayers) as it occurs for some GaAs/AlAs SLs with the zinc blende lattice. It is the reason why our study is focused onto the $\Gamma$ point for dipolar optical electron transitions.
The symmetry of the various structures allows drawing the following conclusions concerning their optical properties.

1) The QWs with the $L 11$ space symmetry and the SLs with the $C_{s}^{1}$ space symmetry have the $\sigma_{v}$ symmetry for the $\Gamma$ point. The subduction procedure of the $\Gamma_{7}$ and $\Gamma_{9}$ IRs of the $C_{6 v}^{4}$ group onto its $L 11$ or $C_{s}^{1}$ subgroup provides the following correspondence

$$
\Gamma_{7}, \Gamma_{9} \rightarrow \Gamma_{3}+\Gamma_{4}
$$

The vector representation is $2 \Gamma_{1}(x, z)+\Gamma_{2}(y)$. Therefore there are bright excitons with the $\Gamma_{1}$ and $\Gamma_{2}$ symmetry. They radiatively recombine with the $x, z$ and $y$ polarizations, respectively. Table 2 displays the Kronecker products of the double-valued IRs of the $\sigma_{v}$ group, together with the allowed polarizations in parentheses for the optical transitions. Note that the transitions between states with the same symmetry are allowed in the $x, z$ polarizations whereas transitions between states with symmetries different one from the other are allowed in the $y$ polarization.

2) The other QWs and SLs have the $C_{2 v}$ symmetry for the $\Gamma$ point. The subduction procedure of the $\Gamma_{7}$ and $\Gamma_{9}$ IRs of the $C_{6 v}^{4}$ group onto its $L 28$ (or 29, or 32, or 33$)$ or $C_{2 v}^{2(\text { or } 5 \text {, or 7) }}$ subgroup provides the following correspondence

$$
\Gamma_{7}, \Gamma_{9} \rightarrow \Gamma_{5}
$$

In the subgroups, there is only a single double-valued IR, the $\Gamma_{5}$ one. Due to our choice of coordinate axes with the $z$ axis parallel to the growth direction, that is the choice usually

Table 2. Kronecker products of $\Gamma$ double-valued IRs in the structures with the $\sigma_{v}$ point group and polarizations in parentheses for the allowed transitions

\begin{tabular}{c|c|c}
\hline IRs & $\Gamma_{3}$ & $\Gamma_{4}$ \\
\hline$\Gamma_{3}$ & $\Gamma_{1}(x, z)$ & $\Gamma_{2}(y)$ \\
$\Gamma_{4}$ & $\Gamma_{2}(y)$ & $\Gamma_{1}(x, z)$
\end{tabular}


made by experimentalists, the vector representation is $\Gamma_{1}(x)+\Gamma_{3}(y)+\Gamma_{4}(z)$ for the type I structures with the $C_{2 v}^{2}$ space or corresponding group. It is $\Gamma_{1}(x)+\Gamma_{3}(z)+\Gamma_{4}(y)$ for the other structures with the $C_{2 v}$ point symmetry. Therefore one has to exchange $y$ and $z$ when giong from the former structures to the latter ones. Keeping the remark in mind we give hereafter results only for the type I structures with the $C_{2 v}^{2}$ group: there are a dark exciton with the $\Gamma_{2}$ symmetry and bright excitons with the $\Gamma_{1}, \Gamma_{3}$, and $\Gamma_{4}$ symmetry that can radiatively recombine with the $x, y$, and $z$ polarization, respectively. The $\Gamma_{5} \times \Gamma_{5}$ Kronecker product is equal to $\Gamma_{1}+\Gamma_{2}+\Gamma_{3}+\Gamma_{4}$ that shows that any carrier transition is allowed in any polarization.

\section{Discussion}

A build-in electric field arising from piezoelectric effect and/or the difference in spontaneous polarizability between the well and barrier materials can exist only in the structures with the $\sigma_{v}$ point symmetry (the type II structures with odd number(s) of monolayers within the slab(s)). Indeed the structures have neither symmetry plane nor glide plane parallel to the substrate surface. Nevertheless, the piezoelectric effect should be weak, if any, in the heterostructures since any monolayer involves the same numbers of cations and anions. Chauveau et al. [17] have grown by MBE onto $R$-plane sapphire some $A$-plane $\mathrm{ZnO}$ layers $1 \mu \mathrm{m}$ thick and two $\mathrm{ZnO} / \mathrm{Zn}_{0.83} \mathrm{Mn}_{0.17} \mathrm{O}$ QWs with well width of 1.6 and $4.1 \mathrm{~nm}$, respectively. The samples have their layer plane parallel to a symmetry plane of the wurtzite lattice (type I structures). The wells exhibit no quantum confined Stark effect, in accordance with their symmetry properties since they have the $C_{2 v}$ point symmetry with a symmetry or glide plane parallel to the layers. In photoluminescence experiments of $\mathrm{ZnO}$ layers, the $A$ and $B$ excitons, in polarization perpendicular to the $c$-axis, and the $C$ exciton, in polarization parallel to the $c$-axis, have been put into evidence (the notations $A, B$, and $C$ for excitons refer to the widely-used ones in bulk wurtzite materials). Therefore, do appear in the spectra only the transitions that are fully allowed i.e., allowed even when the SOI is not taken into account [16]. Note that, when the SOI is taken into account, there exist $B$ and $C$ excitons with the $\Gamma_{1}$ and $\Gamma_{6}$ symmetry, respectively (in our notations $\Gamma_{5}$ and $\Gamma_{6}$ IRs are exchanged in comparison with notations formely used for II-VI wurtzite materials). The excitons can recombine with the polarization parallel and perpendicular to the $c$-axis, respectively but the intensity of the transitions should be weaker since they are allowed only from the SOI.

\section{Conclusion}

A built-in field can exist only in type II structures with odd numbers of monolayers in the wells and in the barriers (for SLs). Analysing the polarization of light in dipolar optical transitions and exciton recombination experiments can help in determining the symmetry of wurtzite-based QWs and SLs with the $c$-axis in the layer plane as well as the parity of the number of monolayers in the slabs.

We thank Dr.Yu. Kitaev and Dr.M. Laugt for very fruitful discussions.

\section{References}

[1] J.S. Im, H. Kollmer, J. Off, A. Sohmer, F. Scholz, A. Hangleiter. Phys. Rev. B 57, R9435 (1998).

[2] N. Grandjean, B. Damilano, S. Dalmasso, M. Leroux, M. Laugt, J. Massies. J. Appl. Phys. 86, 3714 (1999).

[3] M. Leroux, N. Grandjean, J. Massies, B. Gil, P. Lefebvre, P. Bigenwald. Phys. Rev. B 60, 1496 (1999).

[4] C. Morhain, T. Bretagnon, P. Lefebvre, X. Tang, P. Valvin, T. Guillet, B. Gil, T. Taliercio, M. Teisseire-Doninelli, B. Vinter, C. Deparis. Phys. Rev. B 72, 241305 (2005).

[5] M.D. Craven, F. Wu, A. Chakraborty, B. Imer, U.K. Mishra, S.P. DenBaars, J.S. Speck. Appl. Phys. Lett. 84, 1281 (2004).

[6] N. Onojima, J. Suda, T. Kimoto, H. Matsunami. Appl. Phys. Lett. 83, 5208 (2003).

[7] S.H. Lim, D. Shindo. J. Appl. Phys. 88, 5107 (2000).

[8] T. Sasaki, S. Zembutsu. J. Appl. Phys. 61, 2533 (1987); M.D. Craven, S.H. Lim, F. Wu, J.S. Speck, S.P. DenBaars. Appl. Phys. Lett. 81, 469 (2002).

[9] Y. Jun Sun, O. Brandt, U. Jahn, T. Yu Liu, A. Trampert, S. Croneneberg, S. Dhar, K.H. Ploog. J. Appl. Phys. 92, 5714 (2002).

[10] M. McLaurin, T.E. Mates, J.S. Speck. Appl. Phys. Lett. 86, 262104 (2005).

[11] C.Q. Chen, M.E. Gaevski, W.H. Sun, E. Kuokstis, J.P. Zhang, R.S.Q. Fareed, H.M. Wang, J.W. Yang, G. Simin, M.A. Khan, H.P. Maruska, D.W. Hill, M.C. Chou, B. Chai. Appl. Phys. Lett. 91, 3194 (2002).

[12] International tables for crystallography. Vol. E/Ed. Th. Hahn. Reidel, Dordrecht, Holland (2002).

[13] V.P. Smirnov, R.A. Evarestov, A.V. Leko. Fiz. Tverd. Tela 27, 2909 (1985).

[14] E.A. Wood. Bell Syst. Tech. J. 43, 541 (1064).

[15] S.C. Miller, W.F. Love. Tables of irreducible representations of space groups and corepresentations of magnetic space groups. Pruett, Boulder (1967).

[16] P. Tronc, Yu.E. Kitaev, G. Wang, M.F. Limonov, A.G. Panfilov, G. Neu. Phys. Status Solidi B 216, 599 (1999); Yu.E. Kitaev, P. Tronc. Phys. Rev. B 64, 205312 (2001).

[17] J.M. Chauveau, C. Morhain, B. Lo, B. Vinter, P. Vennegues, M. Laught, M. Tesseire-Doninelli, G. Neu. Appl. Phys. A: Mater. Sci. Process. 88, 65 (2007). 